CLINICAL STUDY

\title{
Confirmatory testing in normokalaemic primary aldosteronism: the value of the saline infusion test and urinary aldosterone metabolites
}

\author{
Caroline Schirpenbach ${ }^{1,3}$, Lysann Seiler ${ }^{1}$, Christiane Maser-Gluth ${ }^{4}$, Frank Rüdiger ${ }^{2}$, Christian Nickel $^{2}$, \\ Felix Beuschlein ${ }^{1}$ and Martin Reincke ${ }^{3}$ \\ ${ }^{1}$ Medical Department II and ${ }^{2}$ Medical Department IV, Albert-Ludwigs-University, Freiburg, Germany, ${ }^{3}$ Medizinische Klinik - Innenstadt Klinikum der \\ Ludwig-Maximilians-University, München Ziemssenstr. 1, D-80336 Munich, Germany and ${ }^{4}$ Institute of Pharmacology, Ruprecht-Karls-University, \\ Heidelberg, Germany
}

(Correspondence should be addressed to M Reincke; Email: martin.reincke@med.uni-muenchen.de)

\begin{abstract}
Objective: Primary aldosteronism has recently been recognized as the most frequent cause of secondary hypertension. Since most patients are normokalaemic, differentiation to essential hypertension is challenging. As differentiation by baseline aldosterone/renin ratio may be insufficient, diagnosis should be confirmed by additional tests. However, as most confirmatory tests have been evaluated in hypokalaemic primary aldosteronism only, we reassessed the value of the saline infusion test and $24 \mathrm{~h}$ urinary aldosterone metabolites as confirmatory tests for both normo- and hypokalaemic primary aldosteronism under current antihypertensive medication.

Patients and methods: 25 patients with primary aldosteronism (11 hypokalaemic, 14 normokalaemic), 29 patients with essential hypertension and 47 normotensive subjects were studied. The hypertensives received their usual medication with the exception of spironolactone. All subjects underwent a standard saline infusion test (determination of plasma aldosterone before and after 2.0 liters of isotonic saline for 4 hours i.v.) and collected a $24 \mathrm{~h}$ urine sample for examination of urinary tetrahydroaldosterone and aldosterone-18-glucuronide.

Results: In hypokalaemic primary aldosteronism the saline infusion test showed a reasonable sensitivity (91\%) and specificity (90\%). However, the test failed to differentiate sufficiently between essential hypertension and normokalaemic primary aldosteronism (sensitivity 57\%, specificity 90\%). Similarly, urinary tetrahydroaldosterone had higher sensitivity in hypokalaemic than in normokalaemic primary aldosteronism (sensitivity $64 \%$ vs $36 \%$, specificity $100 \%$ ), whereas for aldosterone- 18 glucuronide, no differences in hypo- and normokalaemic primary aldosteronism were found (sensitivity $45 \%$ and $43 \%$, specificity $100 \%$ ).

Conclusions: These data show that the saline infusion test as an established test in classical hypokalaemic primary aldosteronism is not a reliable test in the normokalaemic variant of the disease. Due to its low accuracy, determination of urinary aldosterone metabolites did not prove useful in confirming either normo- or hypokalaemic patients. We conclude from our data that these tests should not be used as confirmatory testing in the normokalaemic variant of primary aldosteronism.
\end{abstract}

European Journal of Endocrinology 154 865-873

\section{Introduction}

Primary aldosteronism has recently been recognized as the most frequent cause of secondary hypertension, occurring with a prevalence of $5-18 \%$ within the hypertensive population (1-4). Being a potentially curable disease, correct diagnosis is crucial for specific treatment, such as mineralocorticoid antagonist therapy and adrenalectomy (3). Only $10-30 \%$ of patients present with the classical symptoms characterized by hypertension and hypokalaemia, whereas the large majority presents with normal serum potassium, thus showing a milder variant of this disorder (4-6).
The aldosterone/renin ratio is currently the most recommended screening test for primary aldosteronism (3, 6-10). Application of the ratio even under antihypertensive medication is increasingly advocated $(8,11,12)$. A positive test result requires confirmation by functional testing (13), as the specificity of the aldosterone/renin ratio is low $(14,15)$. Various confirmatory tests have been proposed, but an ideal test which is simultaneously simple to perform, sensitive and specific is currently lacking. A common approach to confirming the diagnosis of primary aldosteronism is to demonstrate the insufficient suppression of aldosterone after oral sodium loading, acute saline infusion, and 
administration of captopril or fludrocortisone (3, 6, 13, 16). The fludrocortisone suppression test is regarded by some investigators as the definitive confirmatory test for primary aldosteronism $(3,17)$, but the need for hospital admission due to an associated risk of severe hypokalaemia requiring 6 hourly potassium controls limits its usefulness in practice. The saline infusion test, however, can be performed on an outpatient basis. Based on the principle of a decrease in aldosterone in response to saline-induced renin suppression, the saline infusion test enables a distinction to be made between those patients with essential hypertension and patients with primary aldosteronism who fail to suppress aldosterone sufficiently (18-21). However, all previous studies on the saline infusion test were performed in patients with hypokalaemic primary aldosteronism, whereas the only study investigating both normo- and hypokalaemic patients, based on a previously published aldosterone cut-off value, resulted in the ascription of low sensitivity to the saline infusion test (3).

Another approach in diagnosing primary aldosteronism is to measure aldosterone metabolites in a $24 \mathrm{~h}$ urine sample. This is thought to have the advantage of being independent of circadian variations in plasma levels $(22,23)$. Aldosterone is excreted mainly as tetrahydroaldosterone after metabolization in the liver, and as aldosterone-18-glucuronide produced mainly in the kidney (24).

Appropriate validation of the saline infusion test and urinary aldosterone metabolites as confirmatory tests for normokalaemic primary aldosteronism has not been demonstrated so far. This study, therefore, was designed to reassess the value of the acute saline infusion test and of urinary aldosterone metabolites in patients with confirmed primary aldosteronism receiving their usual hypertensive medication by comparing them with results obtained in normotensive subjects and patients with essential hypertension.

\section{Subjects and methods}

\section{Subjects}

Twenty-five patients with confirmed primary aldosteronism (mean age \pm s.D. $54.8 \pm 10.3$ years; 10 females) and 29 patients with essential hypertension (51.1 \pm 12.2 years; 16 females) referred to our institution over a 15 month period and 47 normotensive subjects (24.1 \pm 2.6 years; 19 females) were enrolled in a prospective controlled trial. Twelve of the twenty-five patients with primary aldosteronism were diagnosed with bilateral adrenal hyperplasia and four had an aldosteroneproducing adenoma. In the remainder, subtype evaluation had not been completed at the end of this study.

With the exception of spironolactone, which was withdrawn at least 6 weeks prior to testing, patients took their regular antihypertensive medication while being studied. Sodium intake was unrestricted. Table 1 shows the clinical data of the patients.

The study protocol was approved by the ethics committee of the University of Freiburg, according to the requirements of the Helsinki Declaration, and informed written consent was obtained from all subjects.

\section{Diagnostic criteria}

All 47 normotensive subjects had a blood pressure below $140 / 90 \mathrm{mmHg}$, no history of hypertension or renal disease, and did not take contraceptives.

Criteria for essential hypertension were blood pressure above 140/90 $\mathrm{mmHg}$, exclusion of secondary forms of hypertension, normal aldosterone/renin ratios (cut-off values according to the mean +2 s.D. of normotensive subjects: aldosterone/renin concentration $<21 \mathrm{pg} / \mathrm{ml}: \mu \mathrm{U} / \mathrm{ml}$, aldosterone/renin activity $<670 \mathrm{pg} / \mathrm{ml}: \mathrm{ng} / \mathrm{ml} / \mathrm{h}$ ), normal serum potassium and normal urinary aldosterone excretion $(<15 \mu \mathrm{g} / 24 \mathrm{~h}$ (41.6 nmol/day)).

The diagnosis of primary aldosteronism was based on biochemical criteria including repeatedly elevated aldosterone/renin ratios (aldosterone/renin concentration $>21 \mathrm{pg} / \mathrm{ml}: \mu \mathrm{U} / \mathrm{ml}$ ), elevated urinary aldosterone excretion ( $>15 \mu \mathrm{g} /$ day), a prior pathological saline infusion test (serum aldosterone at $240 \mathrm{~min}>$ $80 \mathrm{pg} / \mathrm{ml})$, adrenal venous sampling $(n=15)$ and surgery, as described elsewhere $(6,15)$. Diagnosis of the underlying subtype required unequivocal results of posture testing and computed tomography/magnetic resonance imaging (CT/MRI) or a gradient of the aldosterone/cortisol ratio during adrenal venous sampling. The patients were divided according to serum potassium status: patients with previous documented hypokalaemia $(<3.4 \mathrm{mmol} / \mathrm{l})$ were assigned to the hypokalaemic group $(n=11)$, and potassium supplementation was started which normalized potassium in all but three subjects. The remainder with normal potassium were assigned to the normokalaemic group $(n=14)$.

\section{Saline infusion test and $24 \mathrm{~h}$ urine excretion}

All subjects underwent the acute saline infusion test, which was performed by administration of $2000 \mathrm{ml}$ of $0.9 \%$ saline i.v. over $4 \mathrm{~h}$, beginning between 8000 and 9030 h. Subjects remained recumbent during infusion. Before infusion and after $4 \mathrm{~h}$, blood was drawn from a forearm vein for the measurement of plasma aldosterone, plasma renin concentration and plasma renin activity. Blood for measurement of renin activity was kept in crushed ice until centrifuged.

All subjects collected a $24 \mathrm{~h}$ urine sample, which was kept refrigerated until analysis. The urine was collected either the day before or at least 2 days after saline 


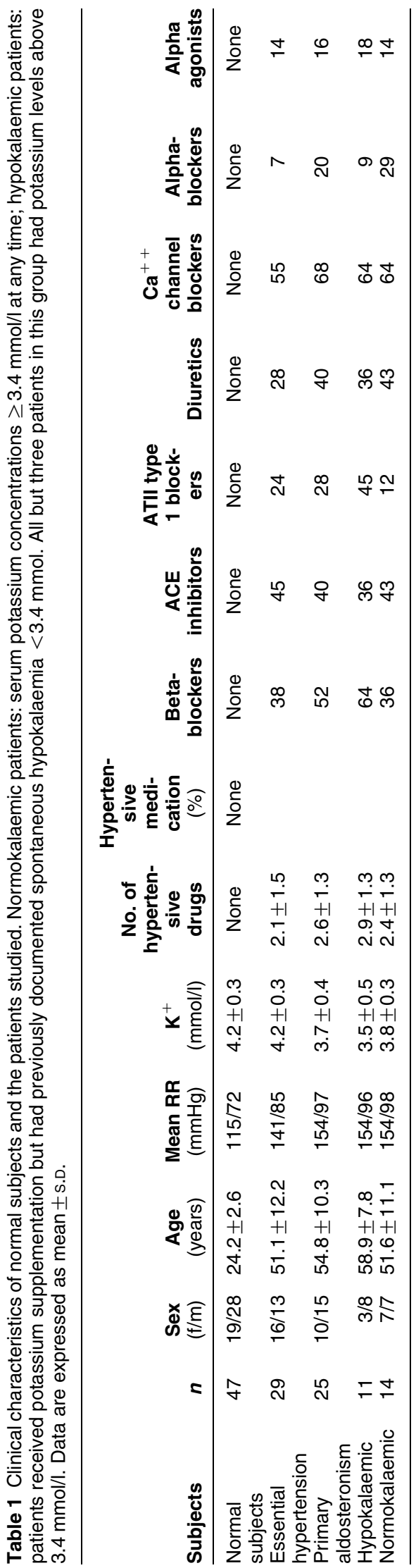

infusion testing. All plasma and urine samples were frozen at $-20^{\circ} \mathrm{C}$ until measured.

\section{Assays}

Plasma aldosterone as well as urinary aldosterone metabolites were determined in the Steroid Laboratory of the University of Heidelberg by radioimmunoassay after extraction and chromatography, as described previously $(25,26)$. Plasma renin concentration was measured on a fully automated immuno-chemiluminescence analyzer (DirectRenin, Nichols Institute Diagnostics, USA). Measurement of plasma renin activity was performed using an angiotensin-I-radioimmunoassay (RENCTK, DiaSorin, Italy). The intraand inter-assay coefficients of variations were $<10 \%$ and $<15 \%$, respectively.

\section{Statistical analysis}

Results are expressed as mean \pm s.D. or s.E.M., as appropriate. Data were compared through use of the unpaired Wilcoxon test, with $P<0.05$ being considered statistically significant. Sensitivity and specificity were calculated using receiver-operating characteristic (ROC) curves. Statistical analysis of the data was performed using Microcal Origin 6.0 (Microcal Origin, Northampton, MA, USA) and SAS 6.12 (SAS, North Carolina, MA, USA).

\section{Results}

\section{Saline infusion test}

Figure 1 shows the baseline hormone characteristics of the patients. Whereas baseline aldosterone concentrations were similar between normal subjects and patients with essential hypertension, baseline renin levels were higher in patients with essential hypertension.

During saline infusion a decrease of mean plasma renin concentration and renin activity was observed in normal controls and patients with essential hypertension (Fig. 1 and Table 2), with the latter still having higher renin levels than the controls. In both groups, the decrease in renin was paralleled by a decrease in mean plasma aldosterone concentrations. In comparison, mean renin levels remained suppressed during saline infusion in primary aldosteronism, whereas aldosterone concentrations decreased after $4 \mathrm{~h}$. In primary aldosteronism, mean aldosterone concentration after saline infusion was significantly higher $(P<0.0001)$ than in the other groups (mean \pm s.D.; primary aldosteronism $14.2 \pm 15.0 \mathrm{ng} / \mathrm{dl}$ (394 \pm $416 \mathrm{pmol} / \mathrm{l})$, essential hypertension $2.1 \pm 1.9 \mathrm{ng} / \mathrm{dl}$ $(58 \pm 53 \mathrm{pmol} / \mathrm{l})$, normal controls $2.3 \pm 1.8 \mathrm{ng} / \mathrm{dl}$ $(64 \pm 50 \mathrm{pmol} / \mathrm{l}))$ (Fig. 2). Calculation of ROC curves 

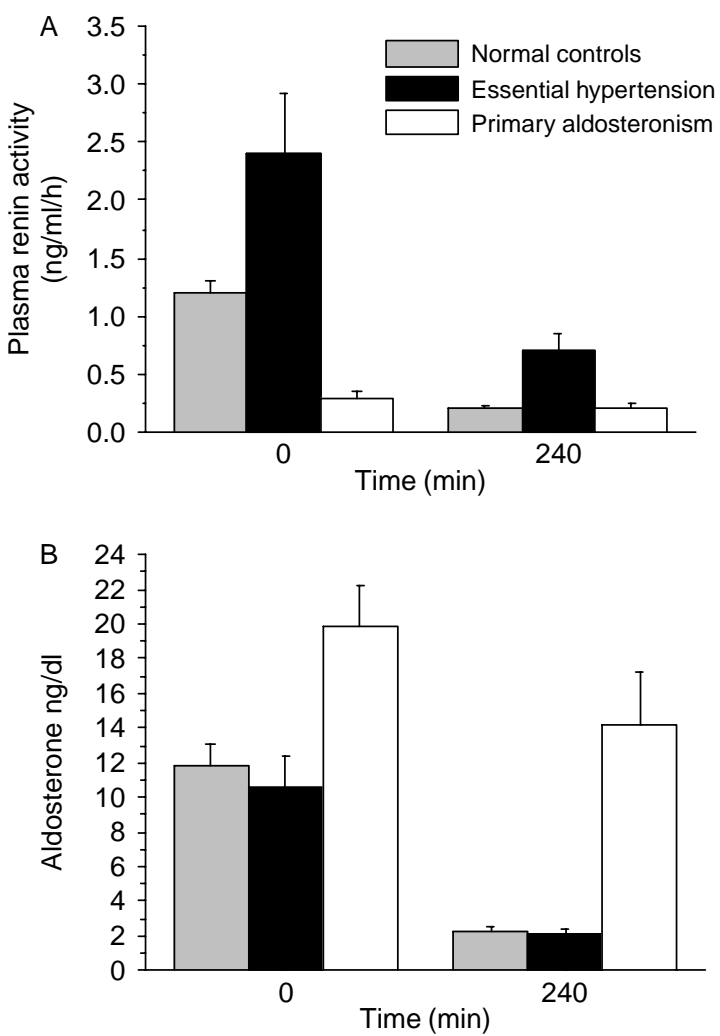

Figure 1 Mean ( \pm S.E.) plasma renin activity $(A)$ and mean $( \pm$ S.E.) plasma aldosterone $(B)$ concentration before $(0 \mathrm{~min})$ and after saline infusion (240 min) in normal subjects, patients with essential hypertension and patients with primary aldosteronism. To convert aldosterone concentrations to pmol/l, multiply by 27.74 .

revealed a sensitivity and specificity of $68 \%$ and $90 \%$, respectively, for differentiation between primary aldosteronism and essential hypertension (cut-off value $5.1 \mathrm{ng} / \mathrm{dl} \quad(141 \mathrm{pmol} / \mathrm{l}))$ (Table 3). However, the response of aldosterone differed highly between normo- and hypokalaemic primary aldosteronism (Fig. 2). Thus, when comparing essential hypertension and hypokalaemic primary aldosteronism, aldosterone after saline infusion had an acceptable specificity $(90 \%)$ and sensitivity $(91 \%)$, whereas the latter was considerably lower in normokalaemic patients ( $57 \%$ sensitivity, $90 \%$ specificity). Analysis of renin and aldosterone levels in the two subgroups of primary aldosterone showed that aldosterone excess seems to be less severe in normokalaemic patients compared to hypokalaemic patients (Table 2). For example, although there were no marked differences between normo- and hypokalaemic patients, plasma renin activity tended to be higher in normokalaemic patients before and after saline infusion than in hypokalaemic patients. Figure 3 shows individual aldosterone curves of patients with essential hypertension and primary aldosteronism, demonstrating that, in general, aldosterone secretion does not appropriately respond to saline infusion in the majority of patients with primary aldosteronism.

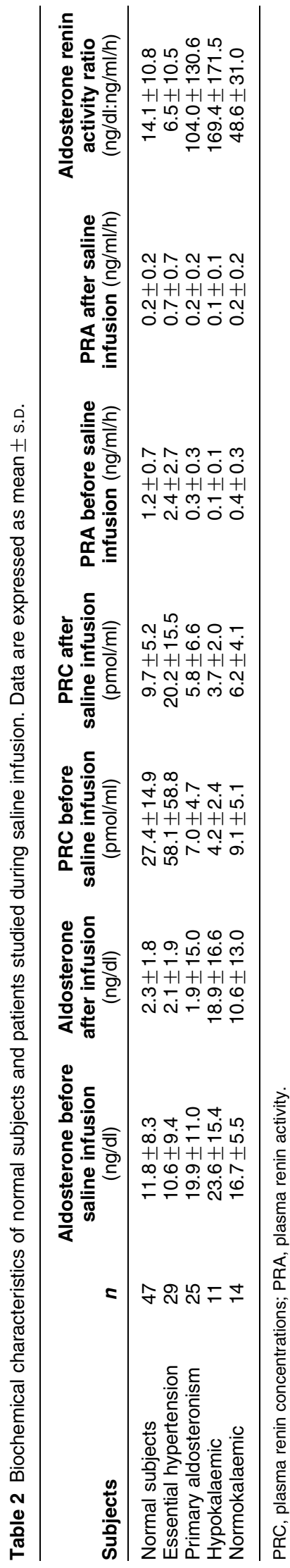




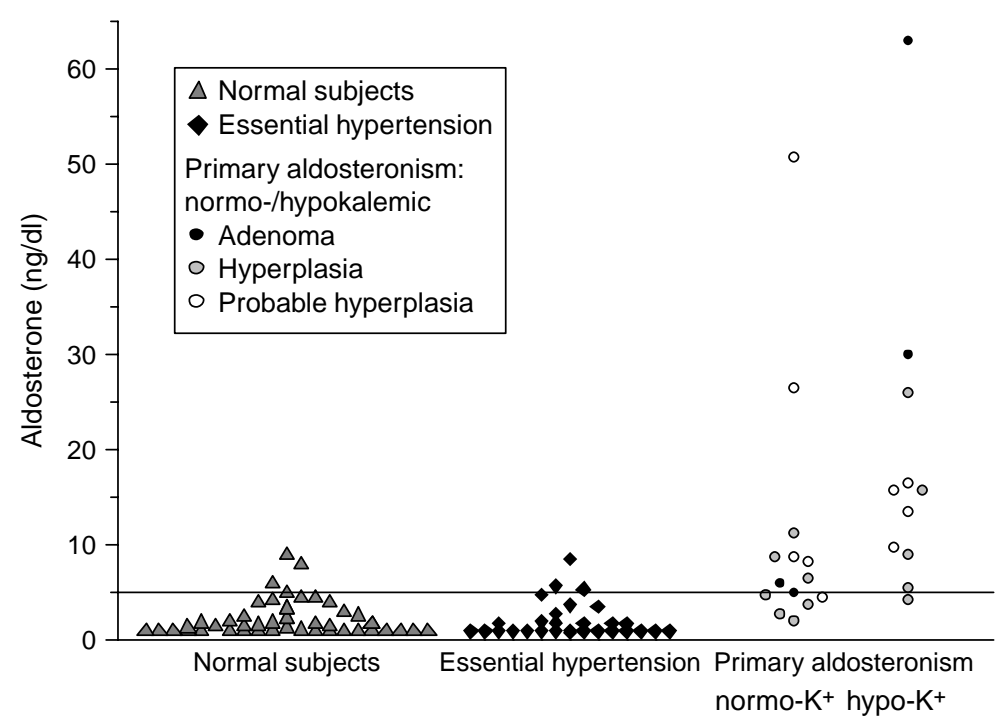

Figure 2 Plasma aldosterone concentrations after saline infusion in normal subjects, patients with essential hypertension and patients with primary aldosteronism. The horizontal line shows the cut-off value of $5.1 \mathrm{ng} / \mathrm{dl}$. Normo-K ${ }^{+}$denotes patients with normal spontaneous potassium, hypo- $\mathrm{K}^{+}$denotes patients with spontaneous hypokalaemia or potassium supplementation due to hypokalaemia. To convert aldosterone concentrations to $\mathrm{pmol} / \mathrm{l}$, multiply by 27.74 .
Blood pressure medication did not have a significant influence on aldosterone concentrations after saline infusion in essential hypertension: aldosterone concentration in patients receiving $12.5 \mathrm{mg}$ hydrochlorothiazide $(n=8)$ was $2.7 \pm 2.6 \mathrm{ng} / \mathrm{dl}$, in patients with angiotensin converting enzyme (ACE)-inhibitors $(n=13) 1.6 \pm 1.0 \mathrm{ng} / \mathrm{dl}$, in patients with ATII type I receptor blockers $(n=7) 2.7 \pm 2.8 \mathrm{ng} / \mathrm{dl}$, and in patients with beta-blockers $(n=11) 1.7 \pm 1.0 \mathrm{ng} / \mathrm{dl}$. Some variability was seen in primary aldosteronism, but it was probably due to sample size and intra-group fluctuations: aldosterone concentration in patients receiving $12.5 \mathrm{mg}$ hydrochlorothiazide $(n=10)$ was $6.4 \pm 3.5 \mathrm{ng} / \mathrm{dl}$, in patients with ACE-inhibitors $(n=10) \quad 19.3 \pm$ $21.1 \mathrm{ng} / \mathrm{dl}$, in patients with ATII type I receptor blockers $(n=7) 13.9 \pm 21.8 \mathrm{ng} / \mathrm{dl}$, and in patients with betablockers $(n=13) 11.3 \pm 8.3 \mathrm{ng} / \mathrm{dl}$.

\section{Aldosterone metabolites}

Mean urinary excretion of tetrahydroaldosterone and aldosterone-18-glucuronide was significantly higher in

Table $3 \mathrm{ROC}$ of plasma aldosterone concentration after saline infusion in patients with normokalaemic and hypokalaemic primary aldosteronism.

\begin{tabular}{lcccc}
\hline $\begin{array}{l}\text { Cut-off } \\
\text { aldoster- } \\
\text { one after } \\
\text { saline } \\
\text { infusion } \\
(\mathrm{ng} / \mathrm{dl})\end{array}$ & $\begin{array}{c}\text { Specificity } \\
(\%)\end{array}$ & $\begin{array}{c}\text { Sensitivity } \\
(\%)\end{array}$ & $\begin{array}{c}\text { Sensitivity } \\
\text { for normo- } \\
\text { kalaemic } \\
\text { patients } \\
(\%)\end{array}$ & $\begin{array}{c}\text { Sensitivity } \\
\text { for hypo- } \\
\text { kalaemic } \\
\text { patients } \\
(\%)\end{array}$ \\
\hline 8.65 & 100 & 52 & 29 & 82 \\
5.8 & 97 & 68 & 57 & 82 \\
5.35 & 93 & 68 & 57 & 82 \\
5.1 & 90 & 72 & 57 & 91 \\
4.7 & 86 & 76 & 64 & 91 \\
4.5 & 86 & 80 & 71 & 91 \\
\hline
\end{tabular}

primary aldosteronism compared to essential hypertension and normal subjects (Table 4). However, substantial overlap was observed between patients with primary aldosteronism and the two other groups (Fig. 4A and B). Since the distribution of the single values suggested these parameters to be suitable for a specific rather than a sensitive test, cut-off values were determined considering a maximum specificity. Thus, cut-off values of $126 \mu \mathrm{g}$ tetrahydroaldosterone/g creatinine and $19.7 \mu \mathrm{g}$ aldosterone-18-glucuronide/g creatinine with a specificity of $100 \%$ revealed a sensitivity of $48 \%$ and $44 \%$, respectively, when separating primary aldosteronism from essential hypertension, with a diagnostic accuracy of $76 \%$ for tetrahydroaldosterone and $74 \%$ for aldosterone-18glucuronide. Whereas sensitivity of tetrahydroaldosterone was found to be better in hypokalaemic than in normokalaemic patients ( $64 \%$ vs $36 \%$, respectively), we did not see a difference between hypokalaemic and normokalaemic patients with respect to aldosterone-18glucuronide ( $45 \%$ vs $43 \%$, respectively).

\section{Discussion}

Primary aldosteronism is meanwhile considered the most common cause of secondary hypertension. However, debate concerning the rationale for screening of primary aldosteronism and concerning the best screening test is continuing $(23,27,28,30)$. Opponents of widespread screening within the hypertensive population argue that the benefit for patients with an elevated aldosterone/renin ratio with respect to outcome measures such as mortality and morbidity has not been shown in randomized controlled trials (31). In addition, screening with the aldosterone/renin ratio has been criticized for a lack of standardization and low 


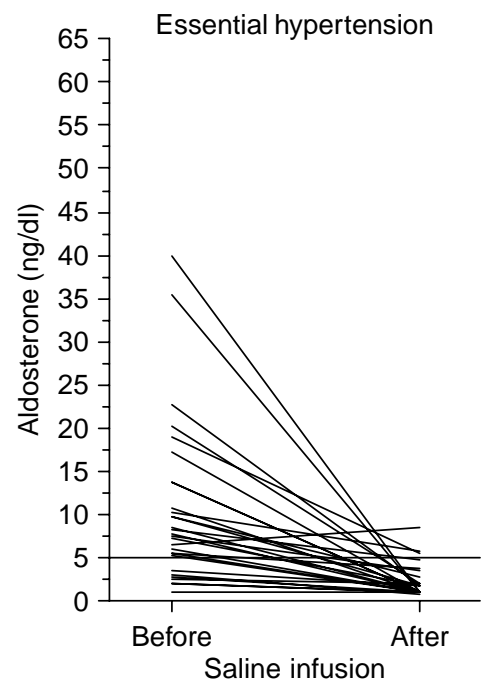

Figure 3 Individual plasma aldosterone concentrations after saline infusion in patients with essential hypertension and patients with primary aldosteronism. To convert aldosterone concentrations to pmol/l, multiply by 27.74 . specificity $(10,28)$. Since differentiation to low renin states such as low renin hypertension and essential hypertension treated with beta-blockers is difficult (32), the putative diagnosis of primary aldosteronism based on a raised aldosterone/renin ratio requires confirmatory testing in order to establish the autonomous character of aldosterone secretion in Conn Syndrome $(3,13)$.

We reassessed the saline infusion test as a confirmatory test for primary aldosteronism. All patients received their usual hypertensive medication with the exception of spironolactone. The rationale for this approach was threefold: (i) in previous studies we and other groups demonstrated that hypertensive medication such as beta-blockers mainly affects renin concentrations but has little, if any, effect on aldosterone levels $(12,32)$; (ii) in the context of an acute volume expansion which is known to strongly suppress aldosterone secretion (33), continuation of antihypertensive medication is even less likely to alter the results of the saline infusion test; and (iii) taking patients with primary aldosteronism off their usual medication is time- and cost intensive, and may harm the patient.

In our study, both patients with essential hypertension and normal controls showed a similar decrease of aldosterone following saline infusion. In addition, there were no significant differences in aldosterone concentrations between patients receiving diuretics, ACE inhibitors, or beta-blockers. Therefore, the normal range in both groups seems to be identical, despite the fact that essential hypertensives used their usual medication. We believe that this is indirect evidence for our hypothesis that hypertensive medication does not influence the aldosterone response to volume loading. However, only a prospective study evaluating the aldosterone response under different blood pressure regimens would provide conclusive evidence for our hypothesis.

In patients with primary aldosteronism, aldosterone also declined during sodium loading but remained significantly higher than in essential hypertension and in normal controls. Thus, aldosterone after saline infusion allowed differentiation between hypokalaemic primary aldosteronism and essential hypertension, with a sensitivity of $91 \%$ and a specificity of $90 \%$ (cut-off value $5.1 \mathrm{ng} / \mathrm{dl}(141 \mathrm{pmol} / \mathrm{l}))$. However, the test proved to be inappropriate for differentiating between normokalaemic primary aldosteronism and patients with essential hypertension ( $57 \%$ sensitivity, 90\% specificity) or normal subjects. The most likely explanation for this phenomenon is that aldosterone excess is less severe and less autonomous in the case of normokalaemic

Table 4 Results of urinary measurements in normotensive subjects, patients with essential hypertension and patients with primary aldosteronism (mean \pm s.D.).

\begin{tabular}{lccc}
\hline Subjects & $\boldsymbol{n}$ & $\begin{array}{c}\text { Tetrahydroaldosterone } \\
(\mu / \mathrm{g} \text { creatinine })\end{array}$ & $\begin{array}{c}\text { Aldosterone-18-glucuronide } \\
(\mu / \mathrm{g} \text { creatinine })\end{array}$ \\
\hline Normal subjects & 47 & $47.3 \pm 20.0$ & $8.3 \pm 4.7$ \\
Essential hypertension & 29 & $57.1 \pm 23.6$ & $8.7 \pm 3.7$ \\
Primary aldosteronism & 25 & $119.0 \pm 49.7^{*} \dagger$ & $18.4 \pm 8.4^{*} \dagger$ \\
Hypokalaemic & 11 & $138.1 \pm 51.8$ & $20.1 \pm 8.1$ \\
Normokalaemic & 14 & $103.9 \pm 44.1$ & $17.1 \pm 8.1$ \\
\hline
\end{tabular}

*Significant difference $(P<0.001)$ between patients with primary aldosteronism and normal subjects.

†Significant difference $(P<0.001)$ between primary aldosteronism and essential hypertension. 

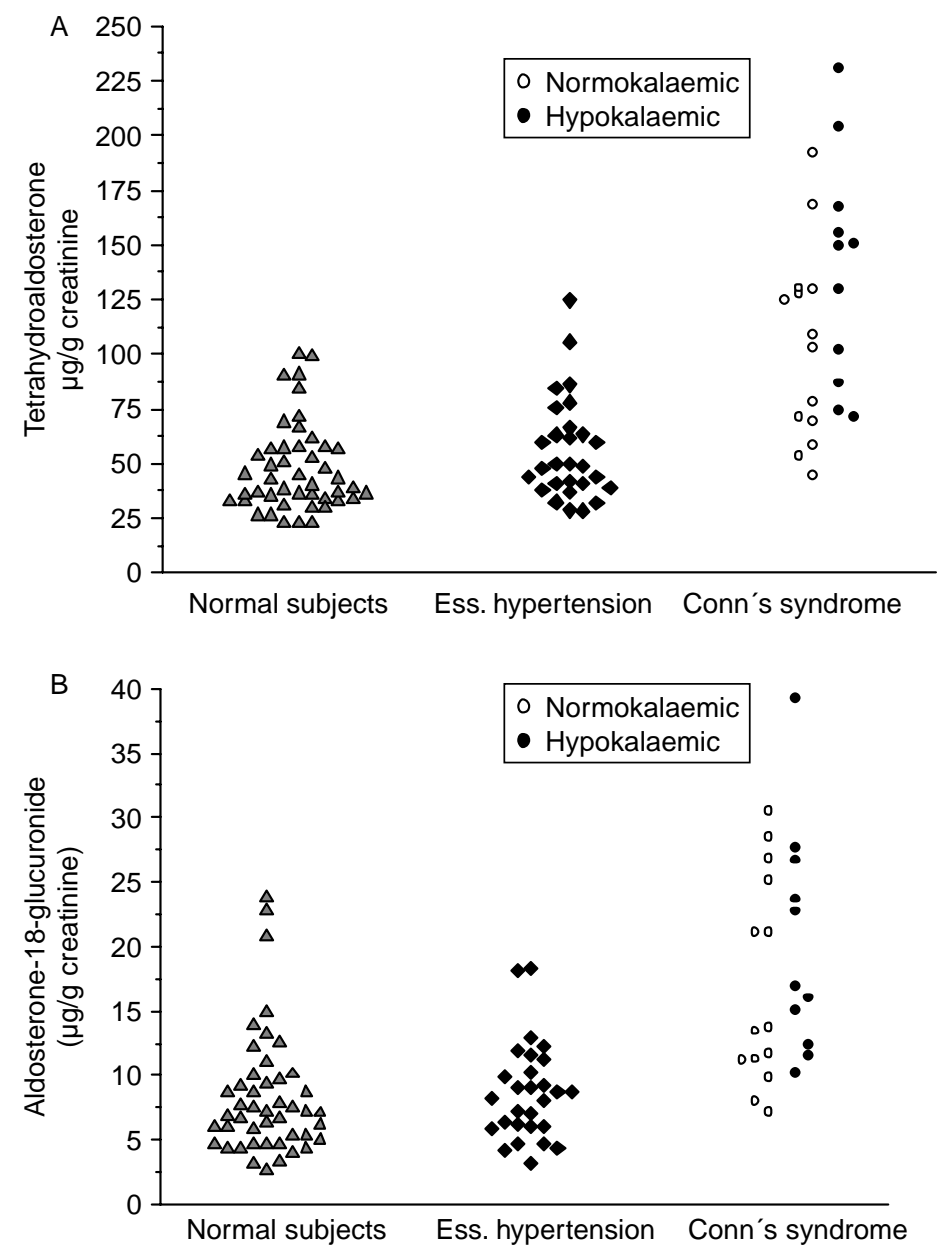

Figure 4 Urinary excretion of aldosterone metabolites. (A) Tetrahydroaldosterone in normotensive subjects, patients with essential hypertension and patients with primary aldosteronism. (B) Aldosterone-18-glucuronide in normotensive subjects, patients with essential hypertension and patients with primary aldosteronism. primary aldosteronism than in the hypokalaemic variant of the disease. This concept is supported by biochemical data in our study showing in normokalaemic primary aldosteronism slightly higher renin levels and lower aldosterone levels both before and after saline infusion. Our data suggest that it is less sensible to use the saline infusion test as a confirmatory test in that the poor detection of normokalaemic primary aldosteronism may lead to inadequate classification of these patients.

Previous studies have employed mainly the saline infusion test to separate Conn's adenoma from essential hypertension $(18,19,21)$ and were performed in hypokalaemic patients. Another study in hypokalaemic aldosteronism investigated the value of the test in differentiating both adenoma and hyperplasia from essential hypertension (34), showing a sensitivity of $96 \%$ and a specificity of $84 \%$, which is comparable to our findings in hypokalaemic patients. In contrast, the low sensitivity of this test was recently demonstrated by Stowasser et al. (3), who reported a sensitivity of 18-30\% in a group of mainly normokalaemic patients with primary aldosteronism. Although these results are partly in line with our data, the extremely low sensitivity in the Stowasser study may also be explained by the use of a previously reported cut-off value of $8 \mathrm{ng} / \mathrm{dl}$ for aldosterone. The ROC analysis performed with our data suggested a lower cut-off value of $5.1 \mathrm{ng} / \mathrm{dl}$ using a highly specific aldosterone assay and thus confirms the results of Kem et al. (21) and Holland (24) who have both suggested a cut-off value of $5 \mathrm{ng} / \mathrm{dl}$. In another study we demonstrated that aldosterone cut-offs after saline infusion varied up to $100 \%$, depending on the assay used (C Shirpenbach et al., unpublished observations). Thus, this reinforces the need to define appropriate cut-off values for each aldosterone assay used.

As another confirmatory test measurement of urinary aldosterone metabolites has been used in order to demonstrate aldosterone excess in primary aldosteronism. Less than $5 \%$ of secreted aldosterone is excreted as free aldosterone into urine, whereas approximately $10 \%$ is excreted as aldosterone-18glucuronide (24). Tetrahydroaldosterone accounts for a higher proportion of aldosterone excretion than aldosterone glucuronide, but nonetheless accounts for only a third of total aldosterone excretion. Some 
aldosterone metabolites are unknown or not determined in clinical practice (36). This is probably the reason why urinary aldosterone determination does not have a better diagnostic value than plasma determinations. Our findings revealed a high overlap for tetrahydroaldosterone and aldosterone-18-glucuronide when comparing primary aldosteronism with essential hypertension. Employing appropriate cut-off values, urinary metabolites proved to have a high specificity for primary aldosteronism, but - owing to high overlap in the lower range of values - a rather low sensitivity. The sensitivity of tetrahydroaldosterone and aldosterone-18-glucuronide did not exceed $48 \%$ and $44 \%$, respectively, for a specificity of $100 \%$. These findings are more or less in line with previous retrospectively collected data by our own group which likewise showed a low sensitivity $(28 \%)$ for tetrahydroaldosterone but a higher one for aldosterone-18-glucuronide $(74 \%)$ at a specificity of $100 \%$ (32). Also, Ulick et al. did not find a significant difference between essential hypertension and primary aldosteronism when measuring urinary tetrahydroaldosterone (35).

Contrary to these findings, Abdelhamid et al. reported a sensitivity and specificity of $95 \%$ and $96 \%$ for tetrahydroaldosterone and $91 \%$ and $71 \%$, respectively, for aldosterone-18-glucuronide (22) in a large study population of 1865 patients using the same assay as in our study. Evaluation of the data differs in that Abdelhamid's group calculated the metabolite concentration as $\mu \mathrm{g}$ /day whereas we expressed the excretion per gram of creatinine in order to correct collection errors. However, this does not seem to be a sufficient explanation for these discrepancies. It is more likely that differences in the design of the studies and diagnostic criteria for primary aldosteronism varied between studies. As a major difference in the study design, Abdelhamid et al. withdrew antihypertensive medication in their patients 2 weeks prior to testing, whereas in our study antihypertensive medication was continued. Thus, in our experience, both tetrahydroaldosterone and aldosterone-18-glucuronide seem to be of limited value for confirming primary aldosteronism under conditions as used in our study.

In summary, both aldosterone concentrations after saline infusion and measurement of urinary aldosterone metabolites did not prove to be useful as a single confirmatory test for normokalaemic primary aldosteronism owing to a high rate of false negatives. Therefore, these tests cannot be recommended as confirmatory tests in patients with suspected normokalaemic primary aldosteronism.

\section{Acknowledgements}

We are grateful to U Bochnig and A Rynk for skilful technical support. We also thank K Muller-Schertler for language editing.

\section{References}

1 Gordon RD, Klemm SA, Stowasser M, Tunny TJ, Storie WJ \& Rutherford JC. How common is primary aldosteronism? Is it the most frequent form of curable hypertension? Journal of Hypertension 199311 (Suppl 5) S310-S311.

2 Lim PO, Rodgers P, Cardale K, Watson AD \& MacDonald TM. Potentially high prevalence of primary aldosteronism in a primarycare population. Lancet 199935340.

3 Stowasser M, Gordon RD, Rutherford JC, Nikwan NZ, Daunt N \& Slater GJ. Diagnosis and management of primary aldosteronism. Journal of the Renin Angiotensin Aldosterone System 20012 156-169.

4 Mulatero P, Stowasser M, Loh KC, Fardella CE, Gordon RD, Mosso L, Gomez-Sanchez CE, Veglio F \& Young WF. Increased diagnosis of primary aldosteronism, including surgically correctable forms, in centers from five continents. Journal of Clinical Endocrinology and Metabolism 200489 1045-1050.

5 Benchetrit S, Bernheim J \& Podjarny E. Normokalemic hyperaldosteronism in patients with resistant hypertension. Israel Medical Association Journal 20024 17-20.

6 Reincke M, Seiler L \& Rump LC. Normokaliämischer primärer Hyperaldosteronismus. Deutsches Ärzteblatt 2003 100 C165-C170.

7 Hiramatsu K, Yamada T, Yukimura Y, Komiya I, Ichikawa K, Ishihara M, Nagata $\mathrm{H}$ \& Izumiyama T. A screening test to identify aldosterone-producing adenoma by measuring plasma renin activity. Archives of Internal Medicine 1981141 1589-1593.

8 Lim PO \& MacDonald TM. Primary aldosteronism, diagnosed by the aldosterone to renin ratio, is a common cause of hypertension. Clinical Endocrinology 200359 427-430.

9 McKenna TJ, Sequeira SJ, Heffernan A, Chambers J \& Cunningham S. Diagnosis under random conditions of all disorders of the renin-angiotensin-aldosterone axis, including primary aldosteronism. Journal of Clinical Endocrinology and Metabolism 199173 952-957.

10 Tiu SC, Choi CH, Shek CC, Ng YW, Chan FK, Ng CM \& Kong AP. The use of aldosterone-renin ratio as a diagnostic test for primary hyperaldosteronism and its test characteristics under different conditions of blood sampling. Journal of Clinical Endocrinology and Metabolism 200590 72-78.

11 Gallay BJ, Ahmad S, Xu L, Toivola B \& Davidson RC. Screening for primary aldosteronism without discontinuing hypertensive medications: plasma aldosterone-renin ratio. American Journal of Kidney Diseases $200137699-705$.

12 Seifarth C, Trenkel S, Schobel H, Hahn EG \& Hensen J. Influence of antihypertensive medication on aldosterone and renin concentration in the differential diagnosis of essential hypertension and primary aldosteronism. Clinical Endocrinology 200257 457-465.

13 Mulatero P, Dluhy RG, Giacchetti G, Boscaro M, Veglio F \& Stewart PM. Diagnosis of primary aldosteronism: from screening to subtype differentiation. Trends in Endocrinology and Metabolism $200516114-119$.

14 Montori VM, Schwartz GL, Chapman AB, Boerwinkle E \& Turner ST. Validity of the aldosterone-renin ratio used to screen for primary aldosteronism. Mayo Clinic Proceedings 200176 $877-882$.

15 Schirpenbach C, Seiler L, Beuschlein F \& Reincke M. Primary aldosteronism: diagnosis and differential diagnosis. Journal of Laboratory Medicine 200428 135-143.

16 Young WF. Primary aldosteronism. Annals of the New York Academy of Sciences 2002970 61-76.

17 Connell JMC \& Fraser R. Primary aldosteronism. In The Oxford Textbook of Endocrinology and Diabetes, pp 791-799. Eds JAH Wass \& SM Shalet. Oxford: Oxford University Press, 2002.

18 Arteaga E, Klein R \& Biglieri EG. Use of the saline infusion test to diagnose the cause of primary aldosteronism. American Journal of Medicine $198579722-728$. 
19 Corvol P, Houde M, Benguigui L, Tonnelier M, Menard J \& Milliez P. Le système rénine-angiotensine-aldostérone chez lez sujets hypertendus. II. Le diagnostic de l'hyperaldostéronisme primaire. La Nouvelle Presse Medicale 19776 2569-2572.

20 Corvol P, Houde M, Menard J \& Milliez P. Le système rénineangiotensine-aldostérone chez les sujets hypertendus. I. Étude analytique chez 124 patients. La Nouvelle Presse Medicale 19776 2483-2487.

21 Kem DC, Weinberger MH, Mayes DM \& Nugent CA. Saline suppression of plasma aldosterone in hypertension. Archives of Internal Medicine $1971 \mathbf{1 2 8} 380-386$.

22 Abdelhamid S, Blomer R, Hommel G, Haack D, Lewicka S, Fiegel P \& Krumme B. Urinary tetrahydroaldosterone as a screening method for primary aldosteronism: a comparative study. American Journal of Hypertension 200316 522-530.

23 Seiler L \& Reincke M. Der Aldosteron-Renin-Quotient bei sekundärer Hypertonie. Herz 200328 686-691.

24 Holland OB. Primary aldosteronism. Seminars in Nephrology 1995 15 116-125.

25 Maser-Gluth C, Reincke M, Allolio B \& Schulze E. Metabolism of glucocorticoids and mineralocorticoids in patients with adrenal incidentalomas. European Journal of Clinical Investigation $2000 \mathbf{3 0}$ (Suppl 3) 83-86.

26 Vecsei P, Abdelhamid S, Mittelstaedt GV, Lichtwald K, Haack D \& Lewicka S. Aldosterone metabolites and possible aldosterone precursors in hypertension. Journal of Steroid Biochemistry 1983 19 345-351.

27 Kaplan NM. Cautions over the current epidemic of primary aldosteronism. Lancet $2001357953-954$.

28 Padfield PL. Prevalence and role of a raised aldosterone to renin ratio in the diagnosis of primary aldosteronism: a debate on the scientific logic of the use of the ratio in practice. Clinical Endocrinology $2003 \mathbf{5 9} 422-426$.
29 Schwartz GL, Chapman AB, Boerwinkle E, Kisabeth RM \& Turner ST. Screening for primary aldosteronism: implications of an increased plasma aldosterone/renin ratio. Clinical Chemistry 200248 1919-1923.

30 Trenkel S, Seifarth C, Schobel H, Hahn EG \& Hensen J. Ratio of serum aldosterone to plasma renin concentration in essential hypertension and primary aldosteronism. Experimental and Clinical Endocrinology and Diabetes 2002110 80-85.

31 Kaplan NM. Caution about the overdiagnosis of primary aldosteronism. The Mayo Clinic Proceedings 200176 875-876.

32 Seiler L, Rump LC, Schulte-Mönting J, Slawik M, Borm K, Pavenstädt H, Beuschlein F \& Reincke M. Diagnosis of primary aldosteronism: value of different screening parameters and influence of antihypertensive medication. European Journal of Endocrinology 2004150 329-337.

33 Dluhy RG, Greenfield M \& Williams GH. Effect of simultaneous potassium and saline loading on plasma aldosterone levels. Journal of Clinical Endocrinology and Metabolism 1977 45 141-146.

34 Holland OB, Brown H, von Kuhnert L, Fairchild C, Risk M \& GomezSanchez CE. Further evaluation of saline infusion for the diagnosis of primary aldosteronism. Hypertension 19846 717-723.

35 Ulick S, Blumenfeld JD, Atlas SA, Wang JZ \& Vaughan ED. The unique steroidogenesis of the aldosteronoma in the differential diagnosis of primary aldosteronism. Journal of Clinical Endocrinology and Metabolism $1993 \mathbf{7 6} 873-878$.

36 Gomez-Sanchez CE \& Holland O. Urinary tetrahydroaldosterone and aldosterone-18-glucuronide excretion in white and black normal subjects and hypertensive patients. Journal of Clinical Endocrinology and Metabolism 198152 214-219.

Received 19 December 2005

Accepted 16 March 2006 